\title{
In-situ permeability from integrated poroelastic reflection coefficients
}

\author{
Karel N. van Dalen, ${ }^{1}$ Ranajit Ghose, ${ }^{1}$ Guy G. Drijkoningen, ${ }^{1}$ and David M. J. Smeulders ${ }^{1}$ \\ Received 3 April 2010; revised 14 May 2010; accepted 19 May 2010; published 23 June 2010.
}

[1] A reliable estimate of the in-situ permeability of a porous layer in the subsurface is extremely difficult to obtain. We have observed that at the field seismic frequency band the poroelastic behavior for different seismic wavetypes can differ in such a way that their combination gives unique estimates of in-situ permeability and porosity simultaneously. This is utilized in the integration of angle- and frequency-dependent poroelastic reflection coefficients in a cost function. Realistic numerical simulations show that the estimated values of permeability and porosity are robust against uncertainties in the employed poroelastic mechanism and in the data. Potential applications of this approach exist in hydrocarbon exploration, hydrogeology, and geotechnical engineering. Citation: van Dalen, K. N., R. Ghose, G. G. Drijkoningen, and D. M. J. Smeulders (2010), In-situ permeability from integrated poroelastic reflection coefficients, Geophys. Res. Lett., 37, L12303, doi:10.1029/2010GL043319.

\section{Introduction}

[2] Reliable information of the distribution of the Darcy permeability $\left(k_{0}\right)$ in a porous layer in the subsurface is critically important in many disciplines, e.g., hydrocarbon exploration, hydrogeology and geotechnical engineering. Permeability can be highly variable, both vertically and horizontally. In general, in-situ $k_{0}$ can hardly be assessed directly [e.g., Ratnam et al., 2005]. The indirect methods are based on empirical relations involving other measured parameters and have large uncertainties. For instance, with the Kozeny-Karman equation and an independent measurement of the porosity $(\phi)$, only an approximate estimate of $k_{0}$ can be obtained.

[3] There have been attempts to estimate in-situ $k_{0}$ from the attenuation of tube waves using poroelastic wave theory [e.g., Burns, 1990]. More recently, seismic body waves have been used for $k_{0}$ estimation employing poroelasticity [de Barros and Dietrich, 2008; Lin et al., 2009]. Poroelasticity predicts a motion of the pore fluid relative to the skeleton as waves propagate through the porous medium. However, at field seismic frequencies $(10-100 \mathrm{~Hz}$ in soft soil, as a conservative range) the relative fluid flow becomes negligible if the porous material is homogeneous or well cemented. The effects of local relative fluid flow become quite substantial if there are heterogeneities like gas inclusions. Goloshubin et al. [2008] have estimated $k_{0}$ from frequency-dependent

\footnotetext{
${ }^{1}$ Faculty of Civil Engineering and Geosciences, Department of Geotechnology, Delft University of Technology, Delft, Netherlands.

Copyright 2010 by the American Geophysical Union. 0094-8276/10/2010GL043319
}

fluid flow and scattering mechanisms.

[4] Strong $k_{0}$ dependence can be observed in the mesoscopic flow mechanisms that can explain the velocity dispersion and attenuation at field seismic frequencies [Pride et al., 2003]. Accounting for mesoscopic flow mechanisms opens the way for exploiting the frequency-dependent seismic reflectivity [Chapman et al., 2006]. The use of seismic reflection data seems particularly advantageous to study the spatial variations of $k_{0}$. A major difficulty, however, arises as many combinations of $k_{0}$ and $\phi$ can explain the observed frequency-dependent velocity and attenuation data, and no unique estimate can be reached.

[5] Here we present the result of an integration of angleand frequency-dependent poroelastic reflection coefficients of different seismic wavetypes at the interface of two fluidsaturated porous layers containing minute quantities of gas. Because the wavetypes behave differently in the $k_{0}-\phi$ domain, their integration in a cost function leads to a unique and reliable estimate for in-situ $k_{0}$ and $\phi$ simultaneously. We first consider a realistic flow mechanism for the field seismic frequencies. Then we illustrate the results through tests on synthetic data, and finally discuss the scope of this approach.

\section{Mesoscopic Flow Mechanism}

[6] For homogeneous porous materials (e.g., glass beads), the wave velocities predicted by Biot's theory are quite accurate [Berryman, 1980]. However, for fluid-saturated natural rocks or sediments, Biot's macroscopic (wavelength scale) flow mechanism cannot simultaneously explain the observed velocity dispersion and attenuation. Recent studies have shown that the major cause of intrinsic attenuation in porous media can be wave-induced local fluid flow due to the presence of mesoscopic (sub-wavelength scale) heterogeneities causing fluid-pressure gradients. Inhomogeneities in the frame structure (e.g., pockets of weakly cemented grains) can be described by the double-porosity theory [Pride et al., 2004]. Inhomogeneities in the fluid (e.g., gas pockets larger than the grain size) can be modeled using an effective plane-wave modulus [White, 1975] or an effective fluid bulk modulus [Smeulders and van Dongen, 1997]. A model for random distributions of inhomogeneities has been proposed by Müller and Gurevich [2005].

[7] In order to investigate the seismic reflection coefficients at the boundary between two porous layers, we consider an unconsolidated near-surface situation made of two layers of water-saturated loose sands containing minute quantities of gas (bubbles). We use the mechanism of Smeulders and van Dongen [1997] and Vogelaar [2009], which uses the Rayleigh-Plesset equation for the gasbubble behavior and is known to provide realistic results [e.g., van Wijngaarden, 1972; Bedford and Stern, 1983]. 
Table 1. Realistic Material Parameters Differing Between the Two Layers

\begin{tabular}{lcccccc}
\hline & $\rho_{s}\left(\mathrm{kgm}^{-3}\right)$ & $\phi$ & $k_{0}\left(\mu \mathrm{m}^{2}\right)$ & $\alpha_{\infty}$ & $G(\mathrm{MPa})$ & $K_{b}(\mathrm{MPa})$ \\
\hline layer 1 & 2500 & 0.4 & 8 & 1.75 & 42.75 & 79.75 \\
layer 2 & 2550 & 0.3548 & 5.5 & 1.91 & 64.80 & 108.00
\end{tabular}

Both sand layers (1: upper layer, 2: lower target layer) have identical grain bulk modulus $K_{s}=36.5 \mathrm{GPa}$, fluid bulk modulus $K_{f}=2.22 \mathrm{GPa}$, fluid viscosity $\eta=0.001 \mathrm{~Pa}$, fluid density $\rho_{f}=1000 \mathrm{kgm}^{-3}$, gas bubble radius $r_{g}=5 \mathrm{~mm}$, gas fraction $s_{g}=0.001$, and gas bulk modulus $K_{g}=142 \mathrm{kPa}$ (air). The two layers differ in shear modulus $G$, bulk modulus $K_{b}$, porosity $\phi$, matrix density $\rho_{s}$, tortuosity $\alpha_{\infty}$, and permeability $k_{0}$ (see Table 1 ).

[8] Using the mesoscopic flow mechanism, seismic wave velocities and attenuations can be computed for both layers. We illustrate in Figure 1 the results for layer 2 as a function of frequency $(f)$. The presence of gas does not affect the shear $(S)$ wave propagation. The fast compressional $\left(P_{1}\right)$ wave is, however, strongly affected by the gas inclusion and shows significant dispersion. The frequency regime where the velocity $c_{P 1}$ sharply changes highly depends on $r_{g}$. For smaller bubbles, as encountered in pressurized marine sediments [Anderson and Hampton, 1980], the dispersive regime shifts towards higher frequencies (Figure 1). At low frequencies, the $P_{1}$-wave attenuation (expressed by the inverse quality factor $\left.\left|Q_{P 1}^{-1}\right|\right)$ shows the typical behavior of sandy sediments [Buchanan, 2006]. Values of $\left|Q_{P 1}^{-1}\right|$ exceeding 0.1 are reported in shallow loose sandy layers [Malagnini, 1996]. Therefore, our considered mechanism offers a realistic description of the seismic dispersion observed in the field.

[9] In the low-frequency limit $c_{P 1}$ only depends on stiffnesses, densities, $\phi$ and $s_{g}$, and it senses $k_{0}$ only at the onset of the dispersive regime. While $\phi$ information is present in both $c_{P 1}$ and $\left|Q_{P 1}^{-1}\right|$, the $k_{0}$ information is mainly implicit in the frequency-dependent attenuation, as $\left|Q_{P 1}^{-1}\right| \propto f / k_{0}$ [Pride et al., 2004].

\section{Permeability From Integration of Reflection Coefficients in a Cost Function}

[10] In this section we present the results of integration of different wavetypes to estimate $k_{0}$ and $\phi$. We use the angle- and frequency-dependent reflection coefficients [e.g., Dutta and Odé, 1983] at the open-pore interface of the two water-saturated gassy sand layers. We incorporate the mesoscopic flow mechanism as discussed above. Regarding reflection coefficients, Johnson et al. [1994] have shown that the coefficients at a fluid/porous-medium interface agree quite well with the experimental results.

[11] In practice, both 3-component seismic (particle velocity) data and the pore-fluid pressure data need to be acquired for different seismic wavetypes $\left(P_{1} P_{1}, P_{1} S V, S V P_{1}, S V S V\right.$, $S H S H)$ at a given location. A reflection event present in these multiple datasets should correspond to a given interface and reflection point, e.g., common midpoint. The data need to be preprocessed to minimize all surface-related effects and various noise, and then decomposed into $P_{1}, S V$ and $S H$ waves. This is not a trivial task for the land data, but recent developments are promising. During all proces-
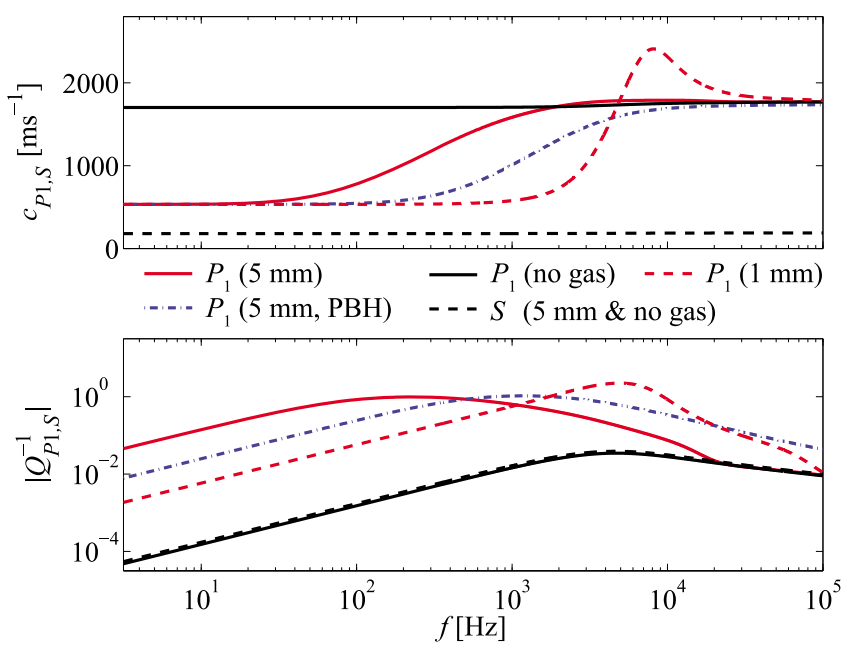

Figure 1. Wave velocities $c_{P 1, S}$ and attenuations $\left|Q_{P 1, S}^{-1}\right|$ in layer 2 for gassy and non-gassy situations [Smeulders and van Dongen, 1997; Vogelaar, 2009]; values of the bubble radius $r_{g}$ are given in brackets. PBH represents the patchy saturation model of Pride et al. [2004], which is used in section 4 .

sing, amplitudes should be preserved. The feasibility of such processing has been reported earlier [e.g., Schalkwijk et al., 2003; Ghose and Goudswaard, 2004; Holvik and Amundsen, 2005].

[12] We assume that all properties of layer 1 are known, and that $k_{0}$ and $\phi$ are the only unknown properties for layer 2 . We calculate the reflection coefficients of various wavetypes $\left(R_{P 1 P 1}, R_{P 1 S V}, R_{S V P 1}, R_{S V S V}\right.$ and $\left.R_{S H S H}\right)$ for varying values of $k_{0}$ and $\phi$ in the target layer (model space: $0.1 \leq k_{0} \leq 100 \leq \mu \mathrm{m}^{2} ; 0.02 \leq \phi \leq 0.7$ ). In Figure 2 we illustrate the behavior of $R_{P 1 P 1}, R_{P 1 S V}$, and $R_{S V S V}$ in the parameter $\left(k_{0}-\phi\right)$ domain. $R_{S V P 1}$ behaves similar to $R_{P 1 S V}$, and $R_{S H S H}$ similar to $R_{S V S V}$. The presence of gas and the resulting high $k_{0}$ sensitivity can only be observed for the reflection coefficients associated with the $P_{1}$ wave, which is due to the influence of gas on $c_{P 1}$ and $\left|Q_{P 1}^{-1}\right|$ only (see Figure 1). Remarkably, the orientation of the contours in the $k_{0}-\phi$ domain is very different, particularly for $R_{P 1 P 1}$ and $R_{P 1 S V}$. This is due to the difference in local (at reflection point) physical behavior of these two wavetypes.

[13] Next, we take advantage of this difference and integrate two different wavetypes by combining their reflection
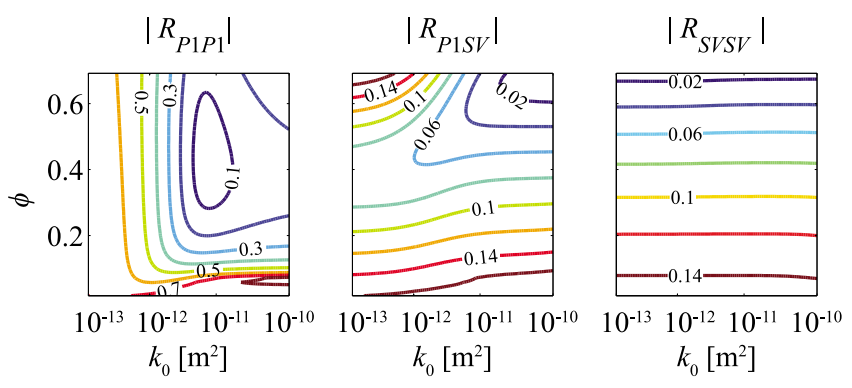

Figure 2. Behavior in the $k_{0}-\phi$ domain of $\left|R_{P 1 P 1}\right|,\left|R_{P 1 S V}\right|$ and $\left|R_{S V S V}\right|$ for $f=50 \mathrm{~Hz}, p=1.110^{-3} \mathrm{~m}^{-1}$. 

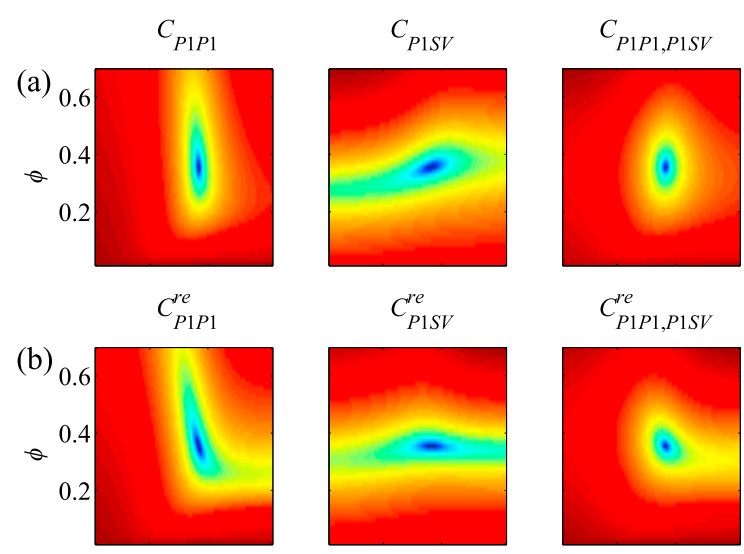

$C_{P 1 P 1}^{i m}$

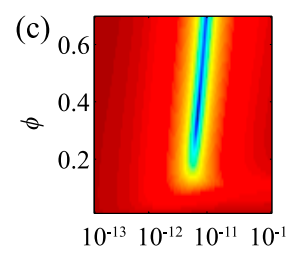

$10^{-13} 10^{-12} 10^{-11}$
$k_{0}\left[\mathrm{~m}^{2}\right]$

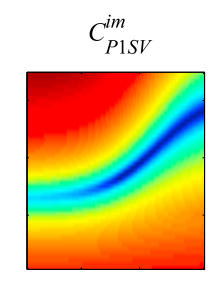

$10^{-13} 10^{-12} 10^{-11}$

$k_{0}\left[\mathrm{~m}^{2}\right]$
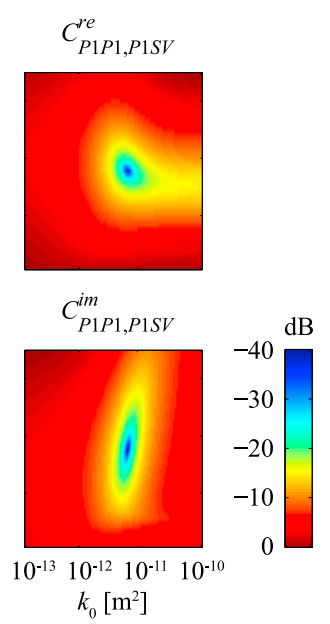

Figure 3. Separate $\left(C_{P 1 P 1}\right.$ and $\left.C_{P 1 S V}\right)$ and integrated $\left(C_{P 1 P 1, P 1 S V}\right)$ cost functions in the $k_{0}-\phi$ domain for (a) total, (b) real, and (c) imaginary part of reflection coefficients.

coefficients in a cost function similar to Ghose and Slob [2006]:

$$
C_{i j, k l}=\left(\frac{\sum_{p, f}\left|\Delta_{i j}\right|^{\beta}}{2\left(\sum_{p, f}\left|\Delta_{i j}\right|^{\beta}\right)_{\max }}+\frac{\sum_{p, f}\left|\Delta_{k l}\right|^{\beta}}{2\left(\sum_{p, f}\left|\Delta_{k l}\right|^{\beta}\right)_{\max }}\right)^{\frac{1}{\beta}},
$$

where $\Delta_{i j}=R_{i j}\left(p, f, k_{0}, \phi\right)-\bar{R}_{i j}(p, f)$ is the difference between model prediction $R_{i j}$, with $j$ denoting the incident wave and $i$ the reflected, and the reflection coefficient $\bar{R}_{i j}$ representing field observation, generated using the true values of $k_{0}$ and $\phi$ (Table 1). Similarly, $\Delta_{k l}$ represents a different wavetype. The cost function involves summation over both the ray parameter $p$ and the frequency $f$. Normalization using the maximum value of each term takes care of the magnitude differences. We use $\beta=2$, but in case of noisy data with a zero mean $\beta=1$ is preferred. The cost function $C_{i j, k l}$ is to be minimized to obtain estimates for $k_{0}$ and $\phi$ in the target layer.

[14] For this numerical test, we adhere to the constraints of near-surface seismic field data in soft soil, viz. the low frequency content and the difficulty in measuring the $P_{1}$-wave dispersion in the field. Therefore, those values are taken only at two discrete frequencies: for $P_{1} 40$ and $100 \mathrm{~Hz}$; for $S 10$ and $50 \mathrm{~Hz} . R_{P 1 S V}$ and $R_{S V P 1}$ have only frequencies that are common to both $P_{1}$ and $S$, hence 40 and $50 \mathrm{~Hz}$. The number of $p$ values is 48 and the corresponding station spacing is $0.75 \mathrm{~m}$. We use only those $p$ values that are related to propagating waves in layer 1 .

[15] In Figure 3 we show the separate cost functions $C_{P 1 P 1}$ and $C_{P 1 S V}$ (individual terms in equation (1)) and the integrated cost function $C_{P 1 P 1, P 1 S V}$. While the separate cost functions do not provide sharp minima, the integrated cost function clearly shows a very sharp minimum (precisely at the correct $k_{0}$ and $\phi$ for layer 2 (see Table 1)). A unique solution for $k_{0}$ and $\phi$ can thus be obtained in the field seismic frequency band. The integration of $C_{P 1 P 1}$ and $C_{P 1 S V}$ offers a good convexity because their individual local minima alignments are nearly orthogonal to each other in the $k_{0}-\phi$ domain. This is due to the underlying physics, as shown in Figure 2: $R_{P 1 P 1}$ has a strong $k_{0}$ sensitivity as it is highly affected by the presence of mesoscopic heterogeneities (gas bubbles), while $R_{P 1 S V}$ is more sensitive to $\phi$. $R_{P 1 S V}$ and $R_{S V P 1}$ are most suitable for integration with $R_{P 1 P 1}$. The other reflection coefficients can only be used for $\phi$ estimation.

[16] The strength of this approach lies in exploiting the physical difference in the poroelastic behavior of the different seismic wavetypes reflected at an interface. Any mechanism of poroelasticity that reliably captures this difference at seismic frequencies will successfully allow such integration. Because the poroelastic reflection coefficients incorporate the effects of both frequency-dependent velocity and attenuation, and angle-dependence provides further constraint, such integration of reflection coefficients is promising. It has been so far impossible to obtain estimates of $k_{0}$ and $\phi$ that individually and simultaneously satisfy the field observations. The present approach provides a solution to this problem.

[17] For pre-critical angles the reflection coefficients have non-zero imaginary parts due to attenuation. Since $k_{0}$ affects mainly the attenuation at the field seismic frequency band, one can intuit that the imaginary part of the reflection coefficient would have the strongest $k_{0}$ sensitivity. In Figure 3 the results for both real and imaginary parts of the reflection coefficients are also illustrated. Because the pre-critical angles are used together with the post-critical ones, the $k_{0}$ sensitivity of the imaginary part decreases. The total reflection coefficient, in this case, offers the best result for integration, as it combines the effect of both real and imaginary parts.

\section{Discussion}

[18] Because all mesoscopic flow mechanisms exhibit strong $k_{0}$ dependence [Pride et al., 2003], reliable estimation of in-situ $k_{0}$ is feasible. To verify the effect of inaccuracy in the observed $\bar{R}_{P 1 P 1}(p, f)$ and $\bar{R}_{P 1 S V}(p, f)$, we have applied to each data point a random perturbation between $\pm 50 \%$ (real and imaginary parts separately). We find that the estimated values of $k_{0}$ and $\phi$ have less than $1 \%$ and $5 \%$ inaccuracy, respectively, indicating the robustness of the method. In order to evaluate the effect of mechanism uncertainty on the estimated values of $k_{0}$ and $\phi$, we have tested two very different mechanisms. We have synthesized $\bar{R}_{P 1 P 1}(p, f)$ and $\bar{R}_{P 1 S V}(p, f)$ using the patchy saturation mechanism of Pride et al. [2004], which also considers mesoscopic gas inclusions in the pore fluid, but employs a simple branching function to connect the low- and highfrequency limits of the frequency-dependent mesoscopic flow. On the other hand, for model predictions, we have, as before, used the mechanism of Smeulders and van Dongen [1997] and Vogelaar [2009]. The dispersive regime and the frequency corresponding to the maximum attenuation are quite different between these two mechanisms (see Figure 1). Next, we have minimized the integrated cost function $C_{P 1 P 1, P 1 S V}$. We find that the effect of mechanism 
uncertainty on the estimated values is small. The value of $\phi$ can be retrieved very accurately ( $<1 \%$ inaccuracy). For $k_{0}$, the error in the retrieved value is less than $25 \%$, which is acceptable for in-situ $k_{0}$. When the frequency restrictions in the field data are slightly relaxed, for instance $300 \mathrm{~Hz}$ for the maximum $P_{1}$-wave frequency and $150 \mathrm{~Hz}$ for the maximum $P_{1} S V$-wave frequency, then the inaccuracy in the $k_{0}$ estimate becomes less than $15 \%$.

[19] In this paper we have considered only two layers. However, the methodology can be adapted to a stack of layers by progressively going downwards. Further, the approach can incorporate more unknown parameters in the lower layer; $r_{g}$ and $s_{g}$ can also be considered unknown. Alternatively, a priori estimates of $r_{g}$ and $s_{g}$ can be obtained from the low-frequency limit of $c_{P 1}$ and the corresponding slope of $\left|Q_{P 1}^{-1}\right|$ [e.g., Pride et al., 2003]. Starting with initial values of $k_{0}$ and $\phi, r_{g}$ and $s_{g}$ can be estimated in an iterative manner.

[20] A reflection coefficient relates to local plane-wave amplitudes and phases. Hence, it contains information about the local $k_{0}$ and it should, therefore, be possible to capture the lateral variation of $k_{0}$ in an otherwise homogeneous layer. The influence of $k_{0}$ fluctuation within a layer [e.g., Müller et al., 2007] can be incorporated in the model reflection coefficients and in decomposition algorithms.

[21] Although the proposed integration of reflection coefficients is powerful, the convexity of the integrated cost function (Figure 2) can also be sensitive to model parameters other than $k_{0}$ and $\phi$. For instance, different combinations of bubble radius $\left(r_{g}\right)$ and gas fraction $\left(s_{g}\right)$ will require different maximum frequency in the data. For $r_{g}=$ $1 \mathrm{~mm}$ and $s_{g}=0.001$, which is typical for marine sediments [Anderson and Hampton, 1980], we find the best $k_{0}$ sensitivity for $C_{P 1 P 1}$ when the maximum $P_{1}$-wave frequency is $400 \mathrm{~Hz}$; this is realistic for marine data. Therefore, one needs to consider a priori if and which reflection coefficients should be integrated, and if the frequency content allows such integration successfully. This can be accomplished through numerical tests.

\section{Conclusions}

[22] We have presented a concept for the estimation of in-situ permeability $\left(k_{0}\right)$ together with porosity $(\phi)$ of a fluid-saturated porous layer containing minute gas inclusions. We have found that reliable and unique estimates can be obtained by minimization of a cost function which integrates local angle- and frequency-dependent poroelastic reflection coefficients. Obtaining simultaneously permeability and porosity estimates from near-surface field seismic data using poroelasticity has so far not been possible. The approach presented here promises a solution to this problem. It takes advantage of the physical difference in the poroelastic behavior of the different seismic wavetypes reflected at the boundary between two porous layers. One needs a poroelastic mechanism that explains data at the field seismic frequencies; we have incorporated a flow mechanism that accounts for mesoscopic inhomogeneity. The validity of the integration approach, however, is not dependent on a specific mechanism. Tests on realistic synthetic data illustrate that the approach is robust against uncertainties in the employed mesoscopic flow mechanism and in the data.
[23] Acknowledgments. This research is supported by The Netherlands Research Centre for Integrated Solid Earth Sciences (ISES).

\section{References}

Anderson, L. A., and L. D. Hampton (1980), Acoustics of gas-bearing sediments. I. Background, J. Acoust. Soc. Am., 67(6), 1865-1889.

Bedford, A., and M. Stern (1983), A model for wave propagation in gassy sediments, J. Acoust. Soc. Am., 73(2), 409-417.

Berryman, J. G. (1980), Confirmation of Biot's theory, Appl. Phys. Lett., 37(4), 382-384.

Buchanan, J. L. (2006), A comparison of broadband models for sand sediments, J. Acoust. Soc. Am., 120(6), 3584-3598.

Burns, D. R. (1990), Acoustic waveform logs and the in-situ measurement of permeability-A review, in Geophysical Applications for Geotechnical Investigations, edited by F. L. Paillet and W. T. Saunders, ASTM Spec. Tech. Publ., 1101, 65-78.

Chapman, M., E. Liu, and X.-Y. Li (2006), The influence of fluid-sensitive dispersion and attenuation on AVO analysis, Geoph. J. Int., 167, 89-105.

de Barros, L., and M. Dietrich (2008), Perturbation of the seismic reflectivity of a fluid-saturated depth-dependent poroelastic medium, J. Acoust. Soc. Am., 123(3), 1409-1420.

Dutta, N. C., and H. Odé (1983), Seismic reflections from a gas-water contact, Geophysics, 48(2), 148-162.

Ghose, R., and J. Goudswaard (2004), Integrating S-wave seismic reflection data and cone-penetration-test data using a multiangle multiscale approach, Geophysics, 69(2), 440-459.

Ghose, R., and E. C. Slob (2006), Quantitative integration of seismic and GPR reflections to derive unique estimates for water saturation and porosity in subsoil, Geophys. Res. Lett., 33, L05404, doi:10.1029/ 2005 GL025376.

Goloshubin, G., D. Silin, V. Vingalov, G. Takkand, and M. Latfullin (2008), Reservoir permeability from seismic attribute analysis, Leading Edge, 27(3), 376-381.

Holvik, E., and L. Amundsen (2005), Elimination of the overburden response from multicomponent source and receiver seismic data, with source designature and decomposition into PP-, PS-, SP-, and SS-wave responses, Geophysics, 70(2), S43-S59.

Johnson, D. L., T. J. Plona, and H. Kojima (1994), Probing porous media with first and second sound. II. Acoustic properties of water-saturated porous media, J. Appl. Phys., 76(1), 115-125.

Lin, L., M. L. Peterson, A. R. Greenberg, and B. A. McCool (2009), In situ measurement of permeability, J. Acoust. Soc. Am., 125(4), EL123EL128.

Malagnini, L. (1996), Velocity and attenuation structure of very shallow soils: Evidence for a frequency-dependent Q, Bull. Seismol. Soc. Am. 86(5), 1471-1486.

Müller, T. M., and B. Gurevich (2005), A first-order statistical smoothing approximation for the coherent wave field in random porous media, J. Acoust. Soc. Am., 117(4), 1796-1805.

Müller, T. M., G. Lambert, and B. Gurevich (2007), Dynamic permeability of porous rocks and its seismic signatures, Geophysics, 72(5), E149E158.

Pride, S. R., et al. (2003), Permeability dependence of seismic amplitudes, Leading Edge, 22(6), 518-525.

Pride, S. R., J. G. Berryman, and J. M. Harris (2004), Seismic attenuation due to wave-induced flow, J. Geophys. Res., 109, B01201, doi:10.1029/ 2003JB002639.

Ratnam, S., K. Soga, and R. W. Whittle (2005), A field permeability measurement technique using a conventional self-boring pressuremeter, Geotechnique, 55(7), 527-537.

Schalkwijk, K. M., C. P. A. Wapenaar, and D. J. Verschuur (2003), Adaptive decomposition of multicomponent ocean-bottom seismic data into downgoing and upgoing P- and S-waves, Geophysics, 68(3), 1091-1102.

Smeulders, D. M. J., and M. E. H. van Dongen (1997), Wave propagation in porous media containing a dilute gas-liquid mixture: Theory and experiments, J. Fluid Mech., 343, 351-373.

van Wijngaarden, L. (1972), One-dimensional flow of liquids containing small gas bubbles, Annu. Rev. Fluid Mech., 4, 369-396.

Vogelaar, B. B. S. A. (2009), Fluid effect on wave propagation in heterogeneous porous media, Ph.D. thesis, Delft Univ. of Technol., Delft, Netherlands.

White, J. E. (1975), Computed seismic speeds and attenuation in rocks with partial gas saturation, Geophysics, 40(2), 224-232.

G. G. Drijkoningen, R. Ghose, D. M. J. Smeulders, and K. N. van Dalen, Department of Geotechnology, Delft University of Technology, PO Box 5048, 2600 GA Delft, Netherlands. (g.g.drijkoningen@tudelft.nl r.ghose@tudelft.nl; d.m.j.smeulders@tudelft.nl; k.n.vandalen@tudelft.nl) 